\title{
Head and Neck Cancer: A High-Risk Population for COVID-19
}

\author{
Flora Yan, $\mathrm{BA}^{1}$ \\ ${ }^{1}$ Affiliation not available
}

April 28, 2020

Flora Yan, $\mathrm{BA}^{1}$; Shaun A. Nguyen, $\mathrm{MD}^{1}$

1: Department of Otolaryngology - Head and Neck Surgery, Medical University of South Carolina.

Word Count: 1,260

Conflicts of Interest: None to Disclose

Corresponding Author:

Flora Yan

Department of Otolaryngology - Head and Neck Surgery

135 Rutledge Avenue, MSC 550, Charleston, SC 29425

843-792-8299

yanf@musc.edu

\section{Abstract}

Since first identified in December of 2019, COVID-19 has disseminated from Wuhan, China rapidly across the globe. $5-8 \%$ of these COVID-19 patients are estimated to become critically ill and will require ICU admission. Predictors of severe/critical ill COVID-19 disease may include increasing age, smoking status, immunosuppression and chronic conditions such as cardiovascular disease, diabetes, hypertension and also cancer. In this brief correspondence, we first describe the outcomes of critically ill patients with and without cancer and extrapolate these findings to the head and neck cancer population.

Dear Dr. Hanna,

Since first identified in December of 2019, severe acute respiratory syndrome coronavirus-2 (SARS-CoV-2) has disseminated from Wuhan, China rapidly across the globe. On March 11 ${ }^{\text {th }}$, 2020 the World Health Organization deemed Coronavirus Disease 2019 (COVID-19) a worldwide pandemic, with the global community in a state of emergency. ${ }^{1}$ As of April 10 $0^{\text {th }}, 2020,1.6$ million COVID-19 cases have been reported worldwide. ${ }^{2}$ Case-fatality rate have ranged from $2 \%$ to $7 \% .{ }^{3}$ Clinically, COVID-19 is initially characterized by a constellation of non-specific symptoms such as cough, fever, and dyspnea. However, this can escalate quickly, with the median time from symptom onset to severe hypoxemia necessitating ICU admission seen to be from 7 to 12 days. ${ }^{4-6}$ It is clear certain populations such as patients with coexisting conditions, older age, an immunocompromised state and a smoking history are at a high risk for severe disease as well as poor outcomes. ${ }^{7}$ Head and neck cancer patients are placed in a vulnerable state and may equally be of highrisk to the consequences of COVID-19, given their immunosuppressed state from cancer and corresponding treatment as well as high prevalence of the aforementioned risk factors. In this correspondence, we aim to discuss sequelae of severe COVID-19 disease, in addition to describing head and neck cancer patients as a high-risk population. 
The majority of COVID-19 cases are of mild severity, however 5-8\% 8,8 of COVID-19 patients may become critically ill, experiencing respiratory failure, septic shock and/or multi-organ failure. This necessitates admission into the intensive care unit (ICU). Two-thirds of these critically ill patients have met criteria for acute respiratory distress syndrome (ARDS) and require advanced respiratory support. The acute severity and rapid progression of COVID-19 is illustrated with over $63 \%$ requiring invasive mechanical ventilation in the first 24 hours of admission. ${ }^{9}$ Mortality of COVID-19 patients in the ICU has been estimated to be $50 \%^{7,10}$. Of these, patients of older age $>70$ years old and with severe comorbidities were seen to have mortality rates of 68 and $59 \%$, respectively. ${ }^{9}$ As defined by the Center for Disease Control's weekly morbidity and mortality report regarding COVID-19, these comorbidities may include diabetes mellitus, chronic lung disease, cardiovascular disease, chronic renal disease, and other chronic disease, of which a history of cancer falls under. ${ }^{11}$

ARDS secondary to COVID-19 requires time on mechanical ventilation than is usually required. Bhatraju et al. ${ }^{7}$ reports a median of 10 days of time on mechanical ventilation before COVID-19 patients were extubated. This is in comparison to 3 to 8 days seen on average for non-COVID related indications for mechanical ventilation. ${ }^{12}$ Even then, most patients are unable to wean off mechanical ventilation, as seen by a tragically high mortality rate of COVID-19 patients on mechanical ventilation (Table 1). The Intensive Care National Audit \& Research Centre (ICNARC) demonstrated a $67.3 \%$ mortality rate of patients receiving advanced (i.e. non-invasive or invasive ventilation, tracheostomy or extracorporeal respiratory support) respiratory support. ${ }^{9}$ Studies from China examining critically ill COVID-19 patients placed on mechanical ventilation have reported mortality rates of $81 \%$ to $97 \% .{ }^{4,5}$ A Seattle-based analysis of critically ill patients on mechanical ventilation saw a comparatively lower mortality rate of $50 \%$, however at the time of this study 3 were still on mechanical ventilation without recovery from COVID-19. ${ }^{7}$ These extraordinary high mortality rates of patients on mechanical ventilation, ranging from $50 \%$ to $97 \%$, may reveal that full intensive care support and life-sustaining therapies still cannot overcome the poor prognosis of certain high-risk populations afflicted by COVID-19. Deterioration despite mechanical ventilation may be confounded by multi-organ system failure. Those who fail mechanical ventilation may be placed on extracorporeal membrane oxygen (ECMO) therapy as end of the line care, however this is often accessible in most hospital systems. In fact, even with substantial cases of critically ill COVID-19 patients, ECMO therapy use has ranged from 6 to $12 \%^{4,5}$

As patients with cancer, especially those in active treatment or in the acute post-treatment phase, are in a particularly immunosuppressed conditions, elucidation of the course of COVID-19 in this patient population is paramount. Liang et al. ${ }^{13}$ describe a cohort of 18 cancer patients $(1$ [6\%] of which with head and neck cancer) having a higher risk of mechanical ventilation or death (39\% vs. 8\%), compared to non-cancer patients. Cancer patients also more rapidly deteriorated, with a median time to a critical event taking 13 days as opposed to 43 days in non-cancer patients.

Multiple other studies have described cancer patients with COVID-19. Desai et al. ${ }^{14}$ performed a metaanalysis of 11 studies describing clinical courses of COVID-19 cases and found a $2 \%$ prevalence of cancer in patients with COVID-19. Desai et al. ${ }^{14}$ also discovered higher risk of severe events for patients recently treated with chemotherapy or surgery in the past 30 days, over non-cancer COVID-19 patients (75\% vs. $43 \%)$.

Zhang et al. ${ }^{15}$ revealed clinical characteristics of 28 COVID-19 infected cancer patients in Wuhan China, of which $3(11 \%)$ had head and neck cancer. Of this cohort, $10(36 \%)$ of patients required mechanical ventilation and $8(29 \%)$ patients died. If assumed these 8 were on maximum respiratory therapy previous to death, a mortality rate of $80 \%$ can be extrapolated and is in line to mortality rates of critically ill non-cancer patients; this, however, is not explicitly validated in the study. Notably, stage IV disease was associated with higher rates of severe events (ICU admission, mechanical ventilation, or death) than stage I-III disease ( $70 \%$ vs $44 \%$ ). Zhang et al. ${ }^{15}$ also revealed patients recently treated with chemotherapy, radiation therapy, and/or immunotherapy in the past 14 days had a 4 -times increased odds of developing a severe event than those who received any treatment $>14$ days.

From this we can observe that 1) prevalence of cancer, active or in remission, in COVID-19 patients is 
higher than in the general population; 2) COVID-19 patients with cancer may deteriorate more rapidly than non-cancer patients 3) active treatment of cancer may be associated with increased risk of severe COVID-19 sequelae than in patients not undergoing treatment; and 4) critically ill COVID-19 patients who have cancer may more likely develop end-stage respiratory failure or death than non-cancer critically ill patients, barring presence of other chronic illnesses. It is difficult to ascertain how cancer patients in remission may far in comparison to the general population, however it is clear patients undergoing active treatment may present as a high-risk population for severe illness following COVID-19 infection. These observations are limited on data provided by retrospective studies of small sample sizes, and thus must be interpreted with caution.

Cancer patients present as a high-risk population for COVID-19 development as well as poorer outcomes. Head and neck cancer patients in particularly may be susceptible to the deleterious effects of not only the viral pathogenesis of COVID-19 itself, but also the long-term psychosocial sequelae of intensive critical care, advanced respiratory treatment and other life-saving measures, all amidst a quarantined environment for a patient population characterized as having twice the suicide risk rate of other cancer patients. Given such high death rates of non-cancer patients on mechanical ventilation, the additive vulnerability from head and neck cancer may make severe or critical ill COVID-19 development quite fatal for our patients. Thus, more attention and perhaps additional testing for patients currently undergoing treatment may be warranted. As described in modified head and neck cancer treatment algorithms ${ }^{16}$, treatment only for advanced head and neck cancers should proceed with full precautions (i.e. COVID testing, PPE) and any possible methods to reduce nosocomial COVID-19 infection is warranted. We hope this correspondence provides insight in the high-risk of head and neck cancer patients for critical illness following COVID-19 infection.

Works Cited

1. World Health Organization. WHO Director-General's opening remarks at the media briefing on COVID19-11 March 2020. 2020; https://www.who.int/dg/speeches/detail/who-director-general-s-opening-remarksat-the-media-briefing-on-covid-19-11-march-2020.

2. Worldometer. COVID-19 coronavirus pandemic. https://www.worldometers.info/coronavirus/. Accessed April 10th, 2020.

3. Onder G, Rezza G, Brusaferro S. Case-Fatality Rate and Characteristics of Patients Dying in Relation to COVID-19 in Italy.JAMA. 2020.

4. Zhou F, Yu T, Du R, et al. Clinical course and risk factors for mortality of adult inpatients with COVID-19 in Wuhan, China: a retrospective cohort study. Lancet. 2020;395(10229):1054-1062.

5. Yang X, Yu Y, Xu J, et al. Clinical course and outcomes of critically ill patients with SARS-CoV-2 pneumonia in Wuhan, China: a single-centered, retrospective, observational study. Lancet Respir Med. 2020.

6. Chen N, Zhou M, Dong X, et al. Epidemiological and clinical characteristics of 99 cases of 2019 novel coronavirus pneumonia in Wuhan, China: a descriptive study. The Lancet.2020;395(10223):507-513.

7. Bhatraju PK, Ghassemieh BJ, Nichols M, et al. Covid-19 in Critically Ill Patients in the Seattle Region - Case Series. New England Journal of Medicine. 2020.

8. Wu Z, McGoogan JM. Characteristics of and Important Lessons From the Coronavirus Disease 2019 (COVID-19) Outbreak in China: Summary of a Report of 72314 Cases From the Chinese Center for Disease Control and Prevention. JAMA. 2020;323(13):1239-1242.

9. ICNARC COVID-19 Study Case Mix Programme Database. ICNARC report on COVID-19 in critica care. 2020; https://www.icnarc.org/About/Latest-News/2020/04/04/Report-On-2249-Patients-CriticallyIll-With-Covid-19.

10. Guan W-j, Ni Z-y, Hu Y, et al. Clinical Characteristics of Coronavirus Disease 2019 in China. New England Journal of Medicine. 2020. 
11. CDC COVID-19 Response Team. Preliminary Estimates of the Prevalence of Selected Underlying Health Conditions Among Patients with Coronavirus Disease 2019 - United States, February 12-March 28, 2020. Morbidity and Mortality Weekly Report. April 3, 2020.

12. Seneff MG, Zimmerman JE, Knaus WA, Wagner DP, Draper EA. Predicting the duration of mechanical ventilation. The importance of disease and patient characteristics. Chest. 1996;110(2):469-479.

13. Liang W, Guan W, Chen R, et al. Cancer patients in SARS-CoV-2 infection: a nationwide analysis in China. Lancet Oncol.2020;21(3):335-337.

14. Desai A, Sachdeva S, Parekh T, Desai R. COVID-19 and Cancer: Lessons From a Pooled Meta-Analysis. JCO Global Oncology.2020(6):557-559.

15. Zhang L, Zhu F, Xie L, et al. Clinical characteristics of COVID-19-infected cancer patients: A retrospective case study in three hospitals within Wuhan, China. Ann Oncol. 2020.

16. Day AT, Sher DJ, Lee RC, et al. Head and neck oncology during the COVID-19 pandemic: Reconsidering traditional treatment paradigms in light of new surgical and other multilevel risks. Oral Oncol.2020:104684.

\section{Hosted file}

HED-20-0517_COVID_Table_1.docx available at https ://authorea.com/users/312328/articles/443222head-and-neck-cancer-a-high-risk-population-for-covid-19 